\title{
AIDS-Related Non-Hodgkin's Lymphoma in Sub-Saharan Africa: Current Status and Realities of Therapeutic Approach
}

\author{
Peter M. Mwamba, ${ }^{1}$ Walter O. Mwanda, ${ }^{1}$ Naftali W. Busakhala, ${ }^{2}$ R. Matthew Strother, ${ }^{3}$ \\ Patrick J. Loehrer, ${ }^{4}$ and Scot C. Remick ${ }^{5}$ \\ ${ }^{1}$ Section of Haematology and Blood Transfusion, Department of Human Pathology, College of Health Sciences, \\ University of Nairobi, Nairobi, Kenya \\ ${ }^{2}$ Departments of Oncology and Pharmacology, Moi University School of Medicine, Eldoret, Kenya \\ ${ }^{3}$ Department of Medicine, Indiana University School of Medicine, Indianapolis, IN 46202, USA \\ ${ }^{4}$ Melvin and Bren Simon Cancer Center, Indiana University, Indianapolis, IN 46202, USA \\ ${ }^{5}$ Mary Babb Randolph Cancer Center, West Virginia University, Morgantown, WV 26506, USA
}

Correspondence should be addressed to Peter M. Mwamba,pmmwamba@yahoo.com

Received 24 February 2012; Revised 30 March 2012; Accepted 7 June 2012

Academic Editor: Jan Delabie

Copyright (C) 2012 Peter M. Mwamba et al. This is an open access article distributed under the Creative Commons Attribution License, which permits unrestricted use, distribution, and reproduction in any medium, provided the original work is properly cited.

Today AIDS-related non-Hodgkin's lymphoma (AR-NHL) is a significant cause of morbidity and mortality in HIV-infected patients the world over, and especially in sub-Saharan Africa. While the overall incidence of AR-NHL since the emergence of combination antiretroviral therapy (cART) era has declined, the occurrence of this disease appears to have stabilized. In regions where access to cART is challenging, the impact on disease incidence is less clear. In the resource-rich environment it is clinically recognized that it is no longer appropriate to consider AR-NHL as a single disease entity and rather treatment of AIDS lymphoma needs to be tailored to lymphoma subtype. While intensive therapeutic strategies in the resource-rich world are clearly improving outcome, in AIDS epicenters of the world and especially in sub-Saharan Africa there is a paucity of data on treatment and outcomes. In fact, only one prospective study of dose-modified oral chemotherapy and limited retrospective studies with sufficient details provide a window into the natural history and clinical management of this disease. The scarcities and challenges of treatment in this setting provide a backdrop to review the current status and realities of the therapeutic approach to AR-NHL in sub-Saharan Africa. More pragmatic and risk-adapted therapeutic approaches are needed.

\section{Introduction}

While the advent of combination antiretroviral therapy (cART) has had a dramatic effect on the clinical manifestations and progression of HIV disease, reduced the incidence of opportunistic infection(s) and AIDS-related malignancies, and improved overall survival in the resource-rich world, the impact of cART scale-up afforded through World Health Organization (WHO) global initiatives and the President's Emergency Plan for AIDS Relief (PEPFAR) in improving access and patient monitoring is less apparent in resource challenged AIDS epicenters of the world and especially Africa [1-6]. The fact remains that the overwhelming majority of HIV-infected individuals in these resource challenged regions are either unaware of their underlying infection and/or go untreated. Thus, the burden of HIV infection and AIDS is greatest in the developing world (95\%) with sub-Saharan Africa harboring essentially two-thirds of the world's population of persons living with HIV/AIDS [7].

Cancer is now a leading cause of morbidity and mortality among individuals living with HIV and AIDS [2, 3, 8-12]. The risk of developing United States (US) Centers for Disease Control AIDS-defining malignancy in HIV-infected subjects is associated with the level of immunodeficiency, especially with Kaposi's sarcoma and non-Hodgkin's lymphoma (NHL); the degree of immunosuppression is less apparent for cervical cancer $[13,14]$. In the US, there is 77 -fold increased risk of developing NHL including Burkitt's lymphoma (BL), which is now the most commonly encountered AIDS-related malignancy and the most common cause of cancer mortality in AIDS patients; however, in Africa it remains the second most common malignant complication following Kaposi's 
sarcoma $[3,8,9]$. Recent estimates in the US also confirm that $5.5 \%$ of diffuse large B-cell lymphoma and $19.4 \%$ of BL cases occurred among persons with AIDS [13]. These lymphomas appear to be more common in males than in females, regardless of antiretroviral use, and there now appears to be a trimodal age-specific incidence pattern for $\mathrm{BL}$ in the US, with the middle-age peak largely composed of cases with AIDS $[8,15]$.

There are limited published reviews on the clinicopathological spectrum of lymphoproliferative diseases encountered in sub-Saharan Africa with or without the backdrop of underlying HIV infection and therapeutic outcome, including both prognostic and predictive indicators. Often these reviews focus more on the adoption of therapeutic approaches from the developed world that may or may not be suitable for the resource challenged setting in sub-Saharan Africa or report clinical experience in patients that are not HIV-infected [16-19]. For instance, in a review from Cape Town, South Africa less than 2\% of 512 consecutively treated lymphoma patients seen at a private sector academic center were HIV-infected [19].

Given this backdrop, it is the intent of our review to primarily focus on the clinicopathological presentation and treatment of AIDS-related non-Hodgkin's lymphoma (AR-NHL) in the resource-limited setting in sub-Saharan Africa. Our report highlights the current realities that the healthcare team is faced with in sub-Saharan Africa and does not recapitulate the data or therapeutic approach from the resource-rich setting. Many obstacles including the diagnosis and management of AR-NHL pose particular problems for clinicians who manage these patients [2025]. These challenges include often unreliable availability of cART, drugs for treatment of opportunistic infection, and poor access to traditional cytotoxic chemotherapy drugs. The prospects for access to even newer agents in the therapeutic armamentarium for lymphoproliferative diseases such as rituximab (Rituxan) and ibritumomab (Zevalin) - CD20directed monoclonal antibodies; bendamustine (Treanda) a new alkylating agent; and brentuximab (Adcetris) - CD30directed monoclonal antibody are nonexistent. Poor access to supportive therapy, which is required during myelotoxic chemotherapy, such as suitable antibiotic coverage, blood products, and colony-stimulating factors (CSFs) all of which are often scarce or nonexistent further complicates patient compliance with therapy, management of associated toxicities, and treatment outcomes especially unacceptably high treatment-related mortality rates when compared to the resource-rich setting. In addition to these, other factors relating to poor referral mechanisms of patients to healthcare facilities, other compliance issues attributable to poverty and ignorance, and late stage clinical presentation all add to the challenges in management of these patients.

\section{The AIDS Epidemic, Antiretroviral; and Cancer Therapy in Sub-Saharan Africa}

The UNAIDS program estimates that 22.5 million adults and children are living in sub-Saharan Africa with HIV
infection/AIDS; the adult seroprevalence rate is on the order of 5\%; and there are 1.3 million deaths annually attributable to HIV disease [7]. South Africa in particular is hardest hit with the largest epidemic of 5.6 million persons [7]. Estimates are that more than a third of HIV-infected individuals residing in sub-Saharan Africa are eligible for antiretroviral therapy receive appropriate cART and patient care. Roll-out of antiretroviral therapy coverage is no doubt improving with an approximate $20 \%$ increase in numbers of patients receiving cART in 2010 when compared to 2009 [7]. This, however, has yet to translate into meaningful decreases in the incidence of AIDS-associated malignant diseases.

Estimates regarding access to or coverage of cytotoxic chemotherapy in sub-Saharan Africa are largely unknown and not published. Access to radiation therapy on the continent is even more scarce; and ample pathology diagnostic capability is often very limited and/or nonexistent in some sub-Saharan African nations [26].

\section{Clinical Presentation of AIDS-Related Non-Hodgkin's Lymphoma}

It was recognized early into the AIDS epidemic that the clinical course of AR-NHL was much more aggressive than patients without HIV infection. In general, AR-NHL is characterized by higher grade (40-60\%), extranodal disease (80\%), advanced clinical stage $(60-70 \%)$ often presenting with B symptoms (i.e., unexplained, fever, night sweats, and weight loss in excess of $10 \%$ of normal body weight), and shortened survival (median 7-8 months) when compared with lymphomas in HIV-seronegative or indeterminate patients $[21,27,28]$. At time of clinical presentation prior to the cART era, the median CD4+ lymphocyte count was $100 / \mu \mathrm{L}$. In the cART era, patients are less immune suppressed with median CD4+ lymphocyte counts ranging between $150-200 / \mu \mathrm{L}$ and higher. In sub-Saharan Africa, it has been recognized for sometime that patients with AIDS-related malignancies do not present with as profound immunosuppression as was observed earlier on in the epidemic in the US [29-31]. It is not uncommon for patients with AIDS-related BL to present with signs and symptoms of tumor lysis syndrome. In addition, the incidence of leptomeningeal involvement at time of diagnosis of ARNHL and over the course of disease appears to be declining as well. This could be attributable to the altered natural history of underlying HIV infection in the cART era and perhaps less predominance of high-grade histologies (offset by increase in intermediate-grade large cell lymphoma) $[21,29]$. Though high-grade histology, especially BL, and lymphomas that harbor EBV, with bone marrow or disease involvement that impinges on or near the CNS such as paranasal sinuses and paraspinal masses are more likely to have leptomeningeal involvement $[21,27,32]$. There remains a clear male predominance in AIDS lymphoma in the US but in other regions of the world most affected by the epidemic such as sub-Saharan Africa, there is nearly an equal distribution of cases in men and women. This is reflective of the predominant heterosexual transmission of HIV infection in Africa. 
Recent studies in Uganda have included a case-control study of NHL (31 adult and 61 pediatrics cases identified between 1994-1998), and two others have reported the clinical characteristics and outcome of pediatric Burkitt's lymphoma (228 cases identified between 1994-2004) and adult NHL (154 cases with sufficient records and excluding BL identified between 2004-2008) in the backdrop of HIV infection for the first time [33-35]. In the case-control study, 92 cases had full phenotyping and documentation of EBV status, which were considered validated [33]. Burkitt's lymphoma and large B-cell lymphomas represented 71\% of adult cases and BL represented $92 \%$ of pediatric cases validated respectively [33]. EBV was present in $35 \%$ of the adult cases and $91 \%$ of pediatric cases. HIV infection was documented in $34 \%$ of adult cases versus $20 \%$ of controls and in $4.9 \%$ of pediatric patients versus $5 \%$ of controls [33]. In another study of children with BL, nearly one-third of cases were HIV-infected, the median age was 6.9 years, and over $60 \%$ were male [34]. HIV-infected children presented with more advanced stage, significant extrafacial (e.g., especially lymphadenopathy), and thoracic disease [34]. The recent large retrospective Ugandan study of 154 cases of NHL in which $32 \%$ were known to be HIV-seropositive underscored the impact of coadministration of cART at time of receiving chemotherapy for lymphoma with outcomes, though dismal, approaching those of HIV-seronegative or indeterminate cases [35]. Interestingly in this series, a third of cases were females; only $60 \%$ of cases had documentation of clinical staging and of these the majority (90\%) presented with stage III or IV disease.

A Kenyan period prevalence study of adult BL in the backdrop of AIDS observed that the proportion of men (60\%) was similar in HIV-seropositive versus seronegative cases; HIV-seropositive cases were significantly older at diagnosis (35 versus 19.5 years); and HIV-seropositive cases uniformly presented with B symptoms and advanced BL accompanied by diffuse lymph node involvement and extranodal presentations as well [36]. What was striking in this study was the complete sparing of peripheral lymph nodes in HIV-seronegative adult BL cases, which is reminiscent of the "typical" pattern of clinical presentation in endemic disease. It was concluded that inclusion of AIDS-related $\mathrm{BL}$ in the differential diagnosis of the adult patient with unexplained fever and lymphadenopathy, which is often associated with Mycobacterium tuberculosis and sexually transmitted diseases in Kenya and other parts of sub-Saharan Africa, warrants consideration. The corollary is that HIV infection is virtually excluded in an adult patient without peripheral lymphadenopathy and biopsy-proven BL [36].

\section{Diagnosis and Staging}

The diagnosis of AR-NHL is established by pathological confirmation of malignant lymphoma on biopsy material of involved lymph node(s), bone marrow, or other extranodal site(s) and should include immunohistochemistry for confirmation of CD20+ B-cell status of the tumor to guide selection of rituximab if this is available; in the majority of resource-limited settings in sub-Saharan Africa this agent is not available $[37,38]$. In the resource challenged setting diagnosis of $\mathrm{BL}$ is often made on fine needle aspiration of peripheral lesions alone and this can present inherent challenges in assurance of diagnosis $[39,40]$. Heightened clinical suspicion upon careful history taking for underlying risk behaviors for acquisition of HIV infection and physical examination for clinical signs and stigmata of HIV disease are critical to properly diagnose and sort out any association of HIV infection and malignant lymphoma. Routine HIV antibody testing is performed in patients with newly diagnosed NHL.

Regardless of setting, patients with AR-NHL are best staged according to the Ann Arbor staging criteria, which is adopted as an international staging classification scheme for NHL [41]. In sub-Saharan Africa clinical staging builds upon careful history and physical examination and incorporates laboratory investigations (including complete blood cell count and differential; serum electrolytes and chemistries, including lactate dehydrogenase where feasible with particular attention to metabolic parameters that are indicative of tumor lysis syndrome); bone marrow aspiration and biopsy; and examination of the cerebrospinal fluid for cytology. In most settings, radiographic studies will be limited to chest radiography and abdominal sonography. Echocardiography is available and usually obtained given the likely use of doxorubicin or other anthracycline-containing combination chemotherapy regimen. Lastly, assessment of HIV infection includes HIV serology, baseline determinations of CD4+ lymphocyte counts, and HIV-1 plasma RNA levels (i.e., viral load).

It is important to specifically comment that reliance on physical examination is all the more important in subSaharan Africa given the relative lack of computed tomography (available in some instances), magnetic resonance imaging, and positron emission tomography (which are generally unavailable) $[21,25,29]$. Although under-staging of patients when compared to western and more resource-rich settings is likely this is, however, balanced by initial presentation at more advanced stages of disease than occurs in developed countries [29]. In this setting, physical examination becomes a reasonable and reliable instrument of assessment. In most situations this is further complemented with accompanying chest radiography, abdominal ultrasonography, bone marrow aspiration biopsy, and cerebrospinal fluid cytology [29].

\section{Historical Overview of Therapeutic Approach to AIDS-Related NHL in the United States}

It is important to comment on the evolution of treatment of AR-NHL in the US or resource-rich setting to put into context the current therapeutic approach in resource-limited sub-Saharan Africa. At the outset of the AIDS epidemic it was readily apparent that patients did not tolerate more aggressive or dose-intensive systemic therapy despite presenting with high-grade tumors including AIDS-associated BL and more advanced stage of disease when compared to HIVseronegative or indeterminate cases of NHL; all patients were 
generally treated in a similar manner regardless of histologic subtype; and prognosis was most dependent on the degree of immunosuppression with patients having demonstrably poorer outcomes with CD4+ lymphocyte counts $<100 / \mu \mathrm{L}$ $[21,27,28,42-44]$. Thus, initial approaches incorporated dose-modified chemotherapeutic strategies, which over the first 15 years of the epidemic proved equally efficacious and markedly less toxic, especially with diminished myelotoxicity $[45,46]$. It was also recognized that infusional versus bolus chemotherapy strategies (e.g., CDE-cyclophosphamide, doxorubicin and etoposide or EPOCH-etoposide, prednisone, vincristine, cyclophosphamide and doxorubicin) yielded better complete response (CR) rates and survival outcomes, though not in randomized or comparative clinical trials [47, 48]. What is also intriguing by the published experience with infusional EPOCH regimen was the strategy of suspension of antiretroviral therapy over the course of chemotherapy to avoid increased risk of drug-drug interactions, potential for increased toxicity, and to enhance overall patient compliance, all of which are appropriate considerations in sub-Saharan Africa [47]. The chemotherapy was also dose-adjusted on the basis of CD4+ lymphocyte count in an attempt to individualize therapy. While this strategy (to suspend antiretroviral therapy) did not result in adverse clinical outcome (i.e., HIV-1 viral load and CD4+ lymphocyte counts returned to baseline by 3 and 12 months, resp.), it should be carefully considered and requires larger, multi-center clinical trial(s) to firmly establish this approach.

The role of rituximab has also been well established in HIV-infected patients with CD20+ B-cell lymphomas despite initial observations (the addition of rituximab to standarddose CHOP led to increased infectious complications and deaths attributable to sepsis) reported by the NCI-sponsored AIDS Malignancy Consortium (AMC 010 study) [49]. Confirmatory studies conducted by the AMC and others have proven the safety of adding rituximab to cytotoxic chemotherapy regimens for AR-NHL, including cases of BL and BL-like subytpes $[27,50]$. Only recently, however, has it been recognized that in the cART era that indeed outcomes are different between subtypes of AIDS lymphoma and that patients with higher grade tumors, and BL in particular, do much worse and need to be treated with more aggressive systemic chemotherapy regimens [51]. It is no longer appropriate to treat all cases of AR-NHL as constituting a single disease entity and into "one therapeutic basket" but rather lymphoma-specific features, especially tumor grade and likely other molecular markers (e.g., CD20+ and IRF4/MUM1-positive immunohistochemistry) need to guide the selection of chemotherapeutic regimens. In the not-too-distant future viral therapeutic targets will likely emerge in the clinic as well [52].

\section{Current Evidence for Treatment of AIDS-Related NHL in Sub-Saharan Africa}

There is scant published data on therapy and clinical outcomes of AR-NHL in sub-Saharan Africa [22, 23]. Table 1 presents clinical data from sub-Saharan Africa in a hierarchal perspective- based on strength of evidence and clinical data from select US clinical trials is summarized for additional insight and comparative purposes [19, 29, 34-36, 45-49, 53]. There is a single prospective clinical trial of dose-modified oral chemotherapy from Kenya and Uganda, and we have selected 4 of the largest retrospective studies that have been published. In the majority of instances, isolated case series or small retrospective studies of AR-NHL in Africa seldom report detailed information on types of chemotherapy administered, safety or toxicity information, and clinical outcomes such as response rates and survival; often data that is published is limited to the clinical presentation and features of disease [22]. On the other hand, published reports of chemotherapy trials in children with endemic BL have emerged, are informative, and have clearly identified the challenges in administering dose-intense chemotherapy in other clinical settings in sub-Saharan Africa [e.g., Malawi and International Society of Paediatric Oncology (SIOP) network], where 1-year event-free survival is $57 \%$ and treatment related mortality is on the order of $30 \%$, which contrasts with at 90\% 1-year EFS rate in Europe and markedly diminished treatment related mortality attributable to the requisite supportive care in the resource-rich environment to sustain children through prolonged periods of dose-intense myelosuppression [54-56]. This experience in children, perhaps more than any other, substantiates the challenges of administering myelosuppressive chemotherapy in settings where resources are scarce and readily translates into the clinical management of patients with AR-NHL. Treatment mortality rates in other disease settings but especially BL, when published, have ranged between $20-66 \%$ in other studies, which is unacceptable in the resource-rich setting [22].

Given this backdrop, in 2009 the first prospective clinical trial of AR-NHL was reported from Kenya and Uganda utilizing a dose-modified oral chemotherapy regimen [29]. It was hypothesized that dose-modified oral chemotherapy using a regimen that had demonstrable activity in ARNHL in the pre-cART era in the US would be efficacious and enhance the therapeutic index [50, 57-59]. Rationale for the 4-drug combination (lomustine-etoposidecyclophosphamide-procarbazine) has been published [57]. What is especially notable is the absence of anthracyclines and hence the avoidance of cardiotoxicity and the presence of agents that cross the blood-brain barrier (lomustine and procarbazine). Corticosteroids were also omitted because of additional immunosuppressive effects and potential tumor growth promoting effects in patients with Kaposi's sarcoma (both endemic and AIDS-related disease) in a region of the world with the highest prevalence and incidence of KSHV infection. Published studies confirmed that dose modification of chemotherapy lessened myelotoxicity without compromising efficacy in the pre-cART era in the US, which provided the departure point to dose modify the oral regimen in sub-Saharan Africa $[45,46]$.

Important outcomes in 49 patients treated on this trial included overall objective response rate of $78 \%$, median event-free and overall survival times of 7.9 months (95\% CI, 3.3 to 13.0 months) and 12.3 months (95\% CI, 4.9 to 32.4 
TABLE 1: Summary of clinical data on the treatment of AIDS-related non-Hodgkin's lymphoma (AR-NHL) in (A) sub-Saharan Africa presented in a hierarchal/strength of evidence approach and (B) the United States presented by year of report (most recent) of prospective clinical trials only. [Abbreviations used: Study/Year-trial number/study sponsor or group and year reported with AMC: AIDS Malignancy Consortium, CWRU: Case Western Reserve University, ECOG: Eastern Cooperative Oncology Group, NCI: National Cancer Institute, and ACTG: AIDS Clinical Trials Group; LD/SD: low dose/standard dose; Nos. Pts.: number patients; BL: Burkitt's lymphoma; ORR: objective response rate $(\mathrm{CR} / \mathrm{PR})$ : complete response/partial response; MST: median survival time, 1-yr : 1-year survival rate and OS: overall survival; and cART: combination antiretroviral therapy.].

\begin{tabular}{|c|c|c|c|c|}
\hline $\begin{array}{l}\text { Regimen (Study/Year) } \\
\text { [Reference] }\end{array}$ & Nos. Pts. & ORR (CR/PR) & Survival & Comment(s) \\
\hline
\end{tabular}

(A) Sub-Saharan Africa Data-Prospective trial (1) and key retrospective studies

Dose-modified oral

chemo. (CWRU

2498/2009) [29]

Uganda Cancer Institute

(NHL study/2011) [35]

154

(32\% HIV+)

512

Stellenbosch University

(NHL study/2010) [19]

(4 HIV+)

Uganda Cancer Institute

(Pediatric BL

study/2009) [34]

University of Nairobi

(BL study/2001) [36]

(31\% HIV+)

796
49

$78 \%$ (CR 58\%/PR
$20 \%)$

No response data provided or types of chemotherapy.

Overall CR range $46-75 \%$ for all subtypes; chemotherapy regimens not reported.

\section{$36 \%$ CR HIV $(+)$} $41 \%$ CR HIV $(-)$ with 29 adult BL (66\% HIV+)
No response data reported or types of chemotherapy.
Only published prospective treatment trial of AR-NHL in sub-Saharan Africa; conducted comparable HIV therapeutic era as ACTG 142 trial [45]; 6\% treatment mortality rate.

Largest retrospective study on NHL including $\mathrm{HIV}(-)$ and $\mathrm{HIV}(+)$ cases ever MST 61 days (13\% 1-yr) reported with treatment and outcome data. Only $60 \%$ had acceptable clinical staging.

Comprehensive retrospective study of spectrum lymphoproliferative disorders

MST 10 mos. (50\% 1-yr) at a major private referral centre in Cape Town. Only 4 cases $(<1 \%)$ were $\operatorname{HIV}(+)$. MST is for the 4 AR-NHL cases.

Comprehensive and largest retrospective MST 11.8 mos. HIV(+) study of pediatric BL in sub-Saharan Not reached $\operatorname{HIV}(-)$ Africa. No details on types of chemotherapy administered.

Among earliest period prevalence and retrospective study that identified 3-fold increase in adult BL cases in the AIDS era. MST is for $\operatorname{HIV}(+)$ BL.
MST 15 wks.

(B) United States Data-Select clinical trials

Concurrent R-EPOCH

versus sequential

R-EPOCH (AMC

034/2010 ) [53]

$\mathrm{R}-\mathrm{CHOP}$ versus $\mathrm{CHOP}$ (AMC 010/2005) [49]

Infusional CDE (ECOG E1494/2004) [48]

Infusional EPOCH

(NCI/2003) [47]

LD and SD CHOP

(AMC 005/2001) [46]
150

$40 \mathrm{LD}$

$23 \mathrm{SD}$

$30 \%$ CR LD

$48 \%$ CR SD

98 LD

41\% CR LD

$52 \%$ CR SD

58\% CR R-CHOP 47\% CR CHOP

45\% CR

$74 \%$ CR

$94 \mathrm{SD}$ 2-yr OS 70\% concurrent versus $67 \%$ sequential

Randomized phase II trial of concurrent versus sequential rituximab with $\mathrm{EPOCH}$ chemotherapy; the primary efficacy endpoint of CR achieved only for the concurrent arm.

Randomized trial among first to use rit-

139 wks. OS R-CHOP 110 uximab; raised concern with higher infecwks. OS CHOP tious deaths on R-CHOP arm; not substantiated in future trials with rituximab.

Among largest studies of a highly active infusional regimen conducted during emergence of cART era.

Dose-adjusted (on basis of CD4+ lymphocyte count) chemotherapy regimen with interruption of cART amongst highest CR and survival reported at time.

Not randomized; 2 consecutive treatment arms established feasibility of concurrent chemotherapy with cART. Median duration of response 9 mos. LD; not reached for SD CHOP.

Largest randomized clinical trial in ARNHL ever conducted; established equivalence of $\mathrm{LD}$ versus SD in pre-cART era. 
months) respectively; and 33\% of patients survived 5 years [29]. The regimen was well tolerated, had modest effects (decline) on CD4+ lymphocyte counts $(P=0.077)$ and had negligible effects on HIV-1 viral replication. Four episodes of febrile neutropenia (5\% of cycles) and three treatmentrelated deaths (6\% mortality rate) occurred. Importantly, there was demonstrable activity in patients with highgrade tumors including 3 cases of verified AIDS-related BL with survivals of $7.2,12.3$ and 14.8 months [29]. It was concluded that dose-modified oral chemotherapy is efficacious, has comparable outcome to that in the US in the pre-cART setting (see data ACTG 142 trial summarized in Table 1) [45], has an acceptable safety profile, and subsequent studies should focus on strategies to optimize combination antiretroviral therapy and chemotherapy and follow-up tissue diagnostic and correlative studies. The NCI-sponsored AIDS Malignancy Consortium is developing a successor trial (AMC 068 protocol in development) of exploring both $\mathrm{CHOP}$ combination chemotherapy and dosemodified oral regimen in sub-Saharan Africa (in Eldoret, Kenya; Harare, Zimbabwe; Johannesburg, South Africa; and Kampala, Uganda) in which all patients will be treated with cART and the oral chemotherapy will be extended from a total course of 12 to 18 weeks (total of three cycles of therapy instead of two as in the original study).

The most thorough and detailed retrospective study reporting clinical outcomes of NHL was published in 2011 also from Uganda [35]. In this study, the median survival of patients presenting with NHL in whom mortality status was confirmed was 61 days; of these $32 \%$ were HIV-seropositive; and median survival among patients with HIV infection receiving antiretroviral therapy was comparable to those without HIV infection [35]. In the majority of instances these patients were treated with standard CHOP combination chemotherapy and dose-adjusted CHOP based on CD4+ lymphocyte count $(<200$ cells $/ \mu \mathrm{L})$ in HIV-infected patients [35]. It is also important to point out in this study that only $60 \%$ of patients were thoroughly clinically staged and there was an enormous rate of loss to follow-up, which highlights the limitations of retrospective studies. Another Uganda study of pediatric $\mathrm{BL}$ reported that while treatment response rates $(\leq 70 \%)$ were similar regardless of HIV-serostatus, median survival (11.79 months) in HIV-infected children was less than HIV-negative/indeterminate children (median survival not reached in these children) [34]. In this report, no details were reported on the types of chemotherapy administered to these children.

Two large retrospective lymphoma studies, one from South Africa and another from Kenya, identified the outcomes of small subsets of HIV-infected patients. A study from a large, private regional university health center in Cape Town reported median survival duration of 10 months in 4 patients with AR-NHL from among 512 patients [19]. In a study from Nairobi of 796 patients with BL; 29 cases of adult BL were identified and two-thirds of these cases were HIVinfected; and, the median survival was 15 weeks [36]. No details were reported on the types of chemotherapy that was administered in either of these studies.
TABLE 2: Formulary of cytotoxic chemotherapy and other agents used for the treatment of NHL and generally available in resourcelimited settings in sub-Saharan Africa. There may be instances where agents are purchased or secured in the private setting (e.g., patients and/or private hospitals) but this falls outside the realities of access to and chemotherapy coverage that is available in large national referral medical centers or regional health centers (e.g., essentially public institutions) in most sub-Saharan Africa nations. Adopted from Orem et al. [22] (Notes: * Supply may be variable; ${ }^{* *}$ generally not available; ${ }^{* * *}$ unavailable.).

\begin{tabular}{lc}
\hline Bleomycin & Leucovorin \\
Cyclophosphamide & Methotrexate \\
Cytarabine & Nitrogen mustard* \\
Dacarbazine* & Prednisone \\
Dactinomycin & Procarbazine* \\
Daunorubicin* & Vinblastine* \\
Doxorubicin & Vincristine \\
Etoposide* & Vinorelbine** \\
Gemcitabine** & Colony-stimulating factors $(\mathrm{CSF})^{* * *}$ \\
Hydroxyurea* $^{* *}$ & Rituximab \\
\hline
\end{tabular}

\section{Departure Point for the Treatment of AR-NHL in Sub-Saharan Africa Based on Current Realities}

Inherent challenges remain in the administration of chemotherapy, supportive care, and follow-up of patients with AR-NHL in sub-Saharan Africa as discussed herein and in more detail elsewhere [21-26, 35, 60]. Table 2 provides a formulary of anticancer agents that are "generally" available in resource limited settings in sub-Saharan Africa. It cannot be overstated that supply of these agents is highly variable and can be interrupted for significant periods of time, which clearly impacts patient follow-up and translates into poor outcomes. Importantly, treatment of AR-NHL has evolved coincident with improvement in antiretroviral therapy. Scale-up of cART in these resource scarce settings will likely have the greatest impact when combined with currently available cytotoxic chemotherapy. There is clear evidence from retrospective and emerging prospective studies that access to cART improves outcomes in patients with AR-NHL.

With this backdrop, in the sub-Saharan Africa CHOP combination chemotherapy (dose-modified in likely majority of instances especially in patients with CD4+ lymphocyte counts $<100$ cells $\mu \mathrm{L}$ or slightly higher) likely represents a standard for patients with AR-NHL based on data from the research-rich world. Extreme caution is advised, however, following the report of retrospective data from Uganda in which the median survival was only 61 days; the majority of these patients received full-dose $\mathrm{CHOP}$ and many required dose interruption, dose reduction, and could not complete therapy [35]. Simply stated, there is no published prospective data on the use of combination CHOP chemotherapy in this setting for patients with AR-NHL. Given this reality, efforts to secure assurance of diagnosis; approaches to exploring oral chemotherapy administration, which undoubtedly consumes less resources and manpower; and dose-modification 
of cytotoxic agents appears prudent in resource-limited settings in Africa. It may also be clinically prudent to initiate antiretroviral therapy for patients once stabilized after their first course of chemotherapy if they are indeed cART naïve at time of AR-NHL diagnosis to limit potential risks of noncompliance with oral cART regimens and the nausea, vomiting seen with chemotherapy and the inherent debility of patients at time of presentation especially those with lymphomatous involvement of the gastrointestinal tract.

\section{Summary and Ways Forward}

In sub-Saharan Africa, in particular, and other developing countries, which bear the greatest burden of AR-NHL, challenges still abound and concerted efforts need to be made to improve patient care in this setting. Clinical research should be prioritized so as to determine the best way to manage this disease by improving diagnostic capability, identifying pragmatic, and better risk-adapted approaches for treatment. Physical examination at time of presentation of patients is an important instrument of measure and disease assessment in this setting. Clinical trials that emerge need to fundamentally improve treatment outcomes that are meaningful to this setting. These treatment trials must be immediately translatable with acceptable safety profiles, costs, and readily adaptable (i.e., any treatment approach cannot overwhelm the healthcare team in terms of its administration) to an environment of often extreme scarcity. The oral chemotherapy regimen reported herein is an excellent departure point for these therapeutic principles in this setting. At the same time there are unprecedented opportunities for translational research to interrogate viral oncogenic pathways given the inherent increased rates of coinfection with viral pathogens such as HIV, EBV, and KSHV among others so unique in this part of the world. This may yield innovative viral-targeted therapeutic strategies and new insights into prevention efforts of AIDS-associated and other viral tumors in this setting altogether.

\section{Acknowledgment}

P. M. Mwamba, W. O. Mwanda, and S. C. Remick are supported in part by NIH grant no.: D43 CA153707. N. W. Busakhala and R. M. Strother are supported in part by NIH grant no.: CA121947.

\section{References}

[1] S. Grabar, V. Le Moing, C. Goujard et al., "Clinical outcome of patients with HIV-1 infection according to immunologic and virologic response after 6 months of highly active antiretroviral therapy," Annals of Internal Medicine, vol. 133, no. 6, pp. 401-I16, 2000.

[2] E. A. Engels, R. J. Biggar, H. I. Hall et al., "Cancer risk in people infected with human immunodeficiency virus in the United States," International Journal of Cancer, vol. 123, no. 1, pp. 187-194, 2008.

[3] A. E. Grulich, M. T. van Leeuwen, M. O. Falster, and C. M. Vajdic, "Incidence of cancers in people with HIV/AIDS compared with immunosuppressed transplant recipients: a meta-analysis," Lancet, vol. 370, no. 9581, pp. 59-67, 2007.

[4] C. Diamond, T. H. Taylor, T. Im, M. Miradi, and H. AntonCulver, "Improved survival and chemotherapy response among patients with AIDS-related non-Hodgkin's lymphoma receiving highly active antiretroviral therapy," Hematological Oncology, vol. 24, no. 3, pp. 139-145, 2006.

[5] C. Diamond, T. H. Taylor, and H. Anton-Culver, "Quality of life, characteristics and survival of patients with HIV and lymphoma," Quality of Life Research, vol. 19, no. 2, pp. 149$155,2010$.

[6] M. Bower, C. Palmieri, and T. Dhillon, "AIDS-related malignancies: changing epidemiology and the impact of highly active antiretroviral therapy," Current Opinion in Infectious Diseases, vol. 19, no. 1, pp. 14-19, 2006.

[7] UNAIDS, "Report on the Global AIDS Epidemic," 2010, http://www.unaids.org/globalreport/.

[8] M. S. Shiels, R. M. Pfeiffer, M. H. Gail et al., "Cancer burden in the HIV-infected population in the United States," Journal of the National Cancer Institute, vol. 103, no. 9, pp. 753-762, 2011.

[9] C. J. Achenbach, S. R. Cole, M. M. Kitahata et al., "Mortality after cancer diagnosis in HIV-infected individuals treated with antiretroviral therapy," AIDS, vol. 25, no. 5, pp. 691-700, 2011.

[10] N. F. Crum, R. H. Riffenburgh, S. Wegner et al., "Comparisons of causes of death and mortality rates among HIV-infected persons: analysis of the pre-, early, and late HAART (Highly Active Antiretroviral Therapy) eras," Journal of Acquired Immune Deficiency Syndromes, vol. 41, no. 2, pp. 194-200, 2006.

[11] F. J. Palella, R. K. Baker, A. C. Moorman et al., "Mortality in the highly active antiretroviral therapy era: changing causes of death and disease in the HIV outpatient study," Journal of Acquired Immune Deficiency Syndromes, vol. 43, no. 1, pp. 2734, 2006.

[12] B. Hasse, B. Ledergerber, H. Furrer et al., "Morbidity and aging in HIV-infected persons: the Swiss HIV cohort study," Clinical Infectious Diseases, vol. 53, no. 11, pp. 1130-1139, 2011.

[13] M. S. Shiels, R. M. Pfeiffer, H. I. Hall et al., "Proportions of kaposi sarcoma, selected non-hodgkin lymphomas, and cervical cancer in the United States occurring in persons with AIDS, 1980-2007," Journal of the American Medical Association, vol. 305, no. 14, pp. 1450-1459, 2011.

[14] M. Frisch, R. J. Biggar, E. A. Engels, and J. J. Goedert, "Association of cancer with AIDS-related immunosuppression in adults," Journal of the American Medical Association, vol. 285, no. 13, pp. 1736-1745, 2001.

[15] S. M. Mbulaiteye, W. F. Anderson, K. Bhatia, P. S. Rosenberg, M. S. Linet, and S. S. Devesa, "Trimodal age-specific incidence patterns for Burkitt lymphoma in the United States, 19732005," International Journal of Cancer, vol. 126, no. 7, pp. 1732-1739, 2010.

[16] F. Sitas, R. Pacella-Norman, H. Carrara et al., "The spectrum of HIV-1 related cancers in South Africa," International Journal of Cancer, vol. 88, no. 3, pp. 489-492, 2000.

[17] G. Sissolak, E. A. Abayomi, and P. Jacobs, "AIDS defining lymphomas in the era of highly active antiretroviral therapy (HAART) - an African perspective," Transfusion and Apheresis Science, vol. 37, no. 1, pp. 63-70, 2007.

[18] A. J. Sasco, A. Jaquet, E. Boidin et al., "The challenge of AIDSrelated malignancies in sub-Saharan Africa," PLoS ONE, vol. 5, no. 1, Article ID e8621, 2010.

[19] G. Sissolak, J. Juritz, D. Sissolak, L. Wood, and P. Jacobs, "Lymphoma-emerging realities in sub-Saharan Africa," 
Transfusion and Apheresis Science, vol. 42, no. 2, pp. 141-150, 2010.

[20] B. N'Galy, S. Bertozzi, and R. W. Ryder, "Obstacles to the optimal management of HIV infection/AIDS in Africa," Journal of Acquired Immune Deficiency Syndromes, vol. 3, no. 4, pp. 430437, 1990.

[21] W. O. Mwanda, C. Banura, E. Katongole-Mbidde et al., "Therapeutic challenges of AIDS-related non-Hodgkin's lymphoma in the United States and East Africa," Journal of the National Cancer Institute, vol. 94, no. 10, pp. 718-732, 2002.

[22] J. Orem, M. W. Otieno, and S. C. Remick, "AIDS-associated cancer in developing nations," Current Opinion in Oncology, vol. 16, no. 5, pp. 468-476, 2004.

[23] J. Orem, M. W. Otieno, and S. C. Remick, "Challenges and opportunities for treatment and research of AIDS-related malignancies in Africa," Current Opinion in Oncology, vol. 18, no. 5, pp. 479-486, 2006.

[24] J. Orem, W. O. Mwanda, C. Banura et al., "Capacity building for the clinical investigation of AIDS malignancy in East Africa," Cancer Detection and Prevention, vol. 29, no. 2, pp. 133-145, 2005.

[25] S. E. Krown, "Cancer in resource-limited settings," Journal of Acquired Immune Deficiency Syndromes, vol. 56, no. 4, pp. 297299, 2011.

[26] W. Wakabi, "Kenya and Uganda grapple with Burkitt lymphoma," The Lancet Oncology, vol. 9, no. 4, p. 319, 2008.

[27] R. Yarchoan, T. S. Uldrick, and R. F. Little, "AIDS-associated lymphomas," in Cancer: Principles and Practice of Oncology, pp. 2099-2112, Lippincott, Williams \& Wilkins, Philadelphia, 9th edition, 2011.

[28] J. L. Ziegler, J. A. Beckstead, and P. A. Volberding, "NonHodgkin's lymphoma in 90 homosexual men. Relation to generalized lymphadenopathy and the acquired immunodeficiency syndrome," New England Journal of Medicine, vol. 311, no. 9, pp. 565-570, 1984.

[29] W. O. Mwanda, J. Orem, P. Fu et al., "Dose-modified oral chemotherapy in the treatment of AIDS-related nonHodgkin's lymphoma in East Africa," Journal of Clinical Oncology, vol. 27, no. 21, pp. 3480-3488, 2009.

[30] J. T. Boerma, A. J. Nunn, and J. A. G. Whitworth, "Mortality impact of the AIDS epidemic: evidence from community studies in less developed countries," AIDS, vol. 12, no. 1, pp. S3-S14, 1998.

[31] S. M. Mbulaiteye, D. M. Parkin, and C. S. Rabkin, "Epidemiology of AIDS-related malignancies an international perspective," Hematology/Oncology Clinics of North America, vol. 17, no. 3, pp. 673-696, 2003.

[32] A. Cingolani, R. Gastaldi, L. Fassone et al., "Epstein-Barr virus infection is predictive of CNS involvement in systemic AIDS-related non-Hodgkin's lymphomas," Journal of Clinical Oncology, vol. 18, no. 19, pp. 3325-3330, 2000.

[33] D. M. Parkin, H. Garcia-Giannoli, M. Raphael et al., "NonHodgkin lymphoma in Uganda: a case-control study," AIDS, vol. 14, no. 18, pp. 2929-2936, 2000.

[34] J. Orem, A. Maganda, E. K. Mbidde, and E. Weiderpass, "Clinical characteristics and outcome of children with burkitt lymphoma in uganda according to HIV infection," Pediatric Blood and Cancer, vol. 52, no. 4, pp. 455-458, 2009.

[35] M. H. Bateganya, J. Stanaway, P. E. Brentlinger et al., "Predictors of survival after a diagnosis of non-Hodgkin lymphoma in a resource-limited setting: a retrospective study on the impact of HIV infection and its treatment," Journal of Acquired Immune Deficiency Syndromes, vol. 56, no. 4, pp. 312-319, 2011.
[36] W. O. Mwanda, S. C. Remick, and C. Whalen, "Adult Burkitt's lymphoma in patients with and without human immunodeficiency virus infection in Africa," International Journal of Cancer, vol. 92, pp. 687-691, 2001.

[37] B. Coiffier, E. Lepage, J. Brière et al., "Chop chemotherapy plus rituximab compared with chop alone in elderly patients with diffuse large-B-cell lymphoma," New England Journal of Medicine, vol. 346, no. 4, pp. 235-242, 2002.

[38] G. Lenz, G. Wright, S. S. Dave, W. Xiao, J. Powell, and H. Zhao, "Stromal gene signatures in large-B-cell lymphomas," New England Journal of Medicine, vol. 359, pp. 2313-2323, 2008.

[39] M. Hummel, S. Bentink, H. Berger et al., "A biologic definition of Burkitt's lymphoma from transcriptional and genomic profiling," New England Journal of Medicine, vol. 354, no. 23, pp. 2419-2430, 2006.

[40] S. S. Dave, K. Fu, G. W. Wright, L. T. Lam, P. Kluin, and E. J. Boerma, "Molecular diagnosis of Burkitt's lymphoma," New England Journal of Medicine, vol. 354, pp. 2431-2442, 2006.

[41] P. P. Carbone, H. S. Kaplan, K. Musshoff, D. W. Smithers, and M. Tubiana, "Report of the committee on Hodgkin's disease staging classification," Cancer Research, vol. 49, pp. 2112-2135, 1979.

[42] J. Stebbing, V. Marvin, and M. Bower, "The evidencebased treatment of AIDS-related Non-Hodgkin's lymphoma," Cancer Treatment Reviews, vol. 30, no. 3, pp. 249-253, 2004.

[43] S. T. Lim and A. M. Levine, "Recent advances in acquired immunodeficiency syndrome (AIDS)-related lymphoma," CA Cancer Journal for Clinicians, vol. 55, no. 4, pp. 229-241, 2005.

[44] N. Mounier, M. Spina, and C. Gisselbrecht, "Modern management of non-Hodgkin lymphoma in HIV-infected patients," British Journal of Haematology, vol. 136, no. 5, pp. 685-698, 2007.

[45] L. D. Kaplan, D. J. Straus, M. A. Testa et al., "Low-dose compared with standard-dose m-BACOD chemotherapy for nonHodgkin's lymphoma associated with human immunodeficiency virus infection," New England Journal of Medicine, vol. 336, no. 23, pp. 1641-1648, 1997.

[46] L. Ratner, J. Lee, S. Tang et al., "Chemotherapy for human immunodeficiency virus-associated non-Hodgkin's lymphoma in combination with highly active antiretroviral therapy," Journal of Clinical Oncology, vol. 19, no. 8, pp. 21712178, 2001.

[47] R. F. Little, S. Pittaluga, N. Grant et al., "Highly effective treatment of acquired immunodeficiency syndrome-related lymphoma with dose-adjusted EPOCH: impact of antiretroviral therapy suspension and tumor biology," Blood, vol. 101, no. 12, pp. 4653-4659, 2003.

[48] J. A. Sparano, S. Lee, M. G. Chen et al., "Phase II trial of infusional cyclophosphamide, doxorubicin, and etoposide in patients with HIV-associated non-Hodgkin's lymphoma: an Eastern Cooperative Oncology Group Trial (E1494)," Journal of Clinical Oncology, vol. 22, no. 8, pp. 1491-1500, 2004.

[49] L. D. Kaplan, J. Y. Lee, R. F. Ambinder et al., "Rituximab does not improve clinical outcome in a randomized phase 3 trial of CHOP with or without rituximab in patients with HIV-associated non-Hodgkin lymphoma: AIDS-Malignancies Consortium Trial 010," Blood, vol. 106, no. 5, pp. 1538-1543, 2005.

[50] J. Orem, P. Fu, A. Ness, W. O. Mwanda, and S. C. Remick, "Oral combination chemotherapy in the treatment of AIDSassociated Hodgkin's disease," East African Medical Journal, vol. 82, no. 9, pp. S144-S149, 2005. 
[51] S. T. Lim, R. Karim, B. N. Nathwani, A. Tulpule, B. Espina, and A. M. Levine, "AIDS-related Burkitt's lymphoma versus diffuse large-cell lymphoma in the pre-highly active antiretroviral therapy (HAART) and HAART eras: significant differences in survival with standard chemotherapy," Journal of Clinical Oncology, vol. 23, no. 19, pp. 4430-4438, 2005.

[52] A. Noy, L. Kaplan, J. Lee, E. Cesarman, and W. Tam, "Modified dose intensive R-CODOX-M/IVAC for HIV-associated Burkitt (BL) (AMC 048) shows efficacy and tolerability, and predictive potential of IRF4/MUM1 expression," in Proceedings of the 13th International Conference on Malignancies in AIDS and Other Acquired Immunodeficiencies, abstract 014, p. 38, Bethesda, Md, USA, 2011.

[53] J. A. Sparano, J. Y. Lee, L. D. Kaplan et al., "Rituximab plus concurrent infusional EPOCH chemotherapy is highly effective in HIV-associated B-cell non-Hodgkin lymphoma," Blood, vol. 115, no. 15, pp. 3008-3016, 2010.

[54] P. B. Hesseling, R. Broadhead, E. Molyneux et al., "Malawi pilot study of burkitt lymphoma treatment," Medical and Pediatric Oncology, vol. 41, no. 6, pp. 532-540, 2003.

[55] P. Hesseling, R. Broadhead, E. Mansvelt et al., "The 2000 burkitt lymphoma trial in Malawi," Pediatric Blood and Cancer, vol. 44, no. 3, pp. 245-250, 2005.

[56] P. B. Hesseling, E. Molyneux, F. Tchintseme et al., "Treating Burkitt's lymphoma in Malawi, Cameroon, and Ghana," The Lancet Oncology, vol. 9, no. 6, pp. 512-513, 2008.

[57] S. C. Remick, J. J. McSharry, B. C. Wolf et al., "Novel oral combination chemotherapy in the treatment of intermediategrade and high-grade AIDS-related non-Hodgkin's lymphoma," Journal of Clinical Oncology, vol. 11, no. 9, pp. 16911702, 1993.

[58] S. C. Remick, N. Sedransk, R. Haase et al., "Oral combination chemotherapy in the management of AIDS-related lymphoproliferative malignancies," Drugs, vol. 58, no. 3, pp. 99-107, 1999.

[59] S. C. Remick, N. Sedransk, R. F. Haase et al., "Oral combination chemotherapy in conjunction with filgrastim (G-CSF) in the treatment of AIDS-related non-Hodgkin's lymphoma: evaluation of the role of G-CSF; Quality-of-life analysis and long-term follow-up," American Journal of Hematology, vol. 66, no. 3, pp. 178-188, 2001.

[60] R. J. Lingwood, P. Boyle, A. Milburn et al., "The challenge of cancer control in Africa," Nature Reviews Cancer, vol. 8, no. 5, pp. 398-403, 2008. 


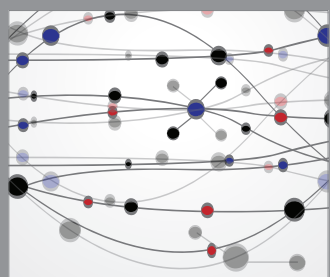

The Scientific World Journal
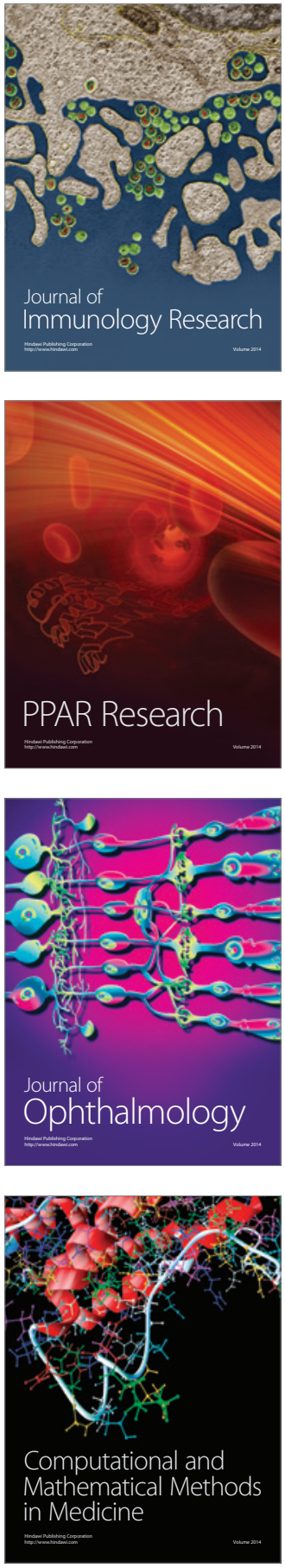

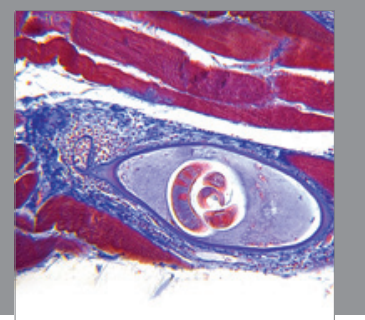

Gastroenterology

Research and Practice
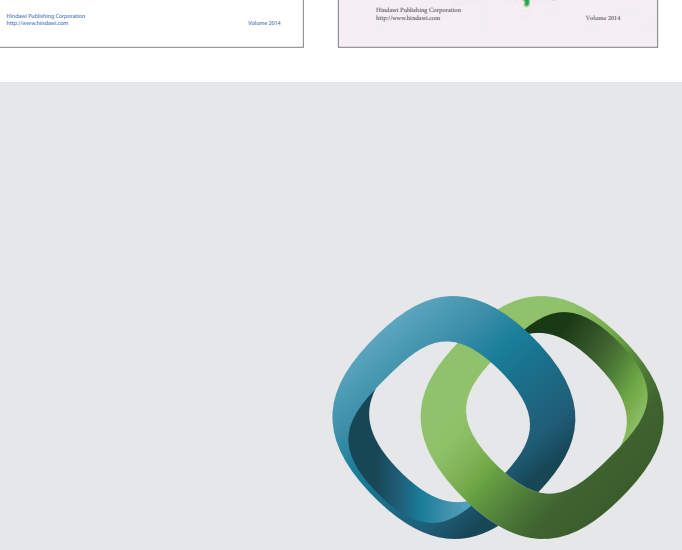

\section{Hindawi}

Submit your manuscripts at

http://www.hindawi.com
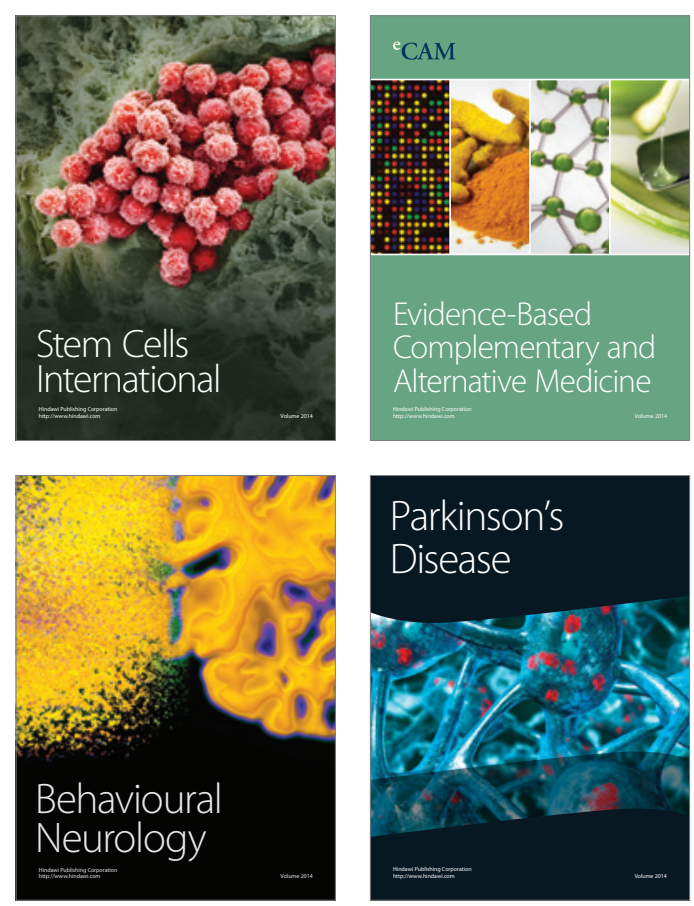

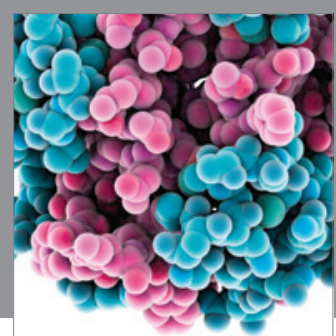

Journal of
Diabetes Research

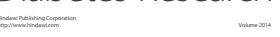

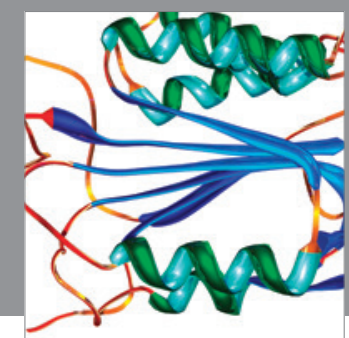

Disease Markers
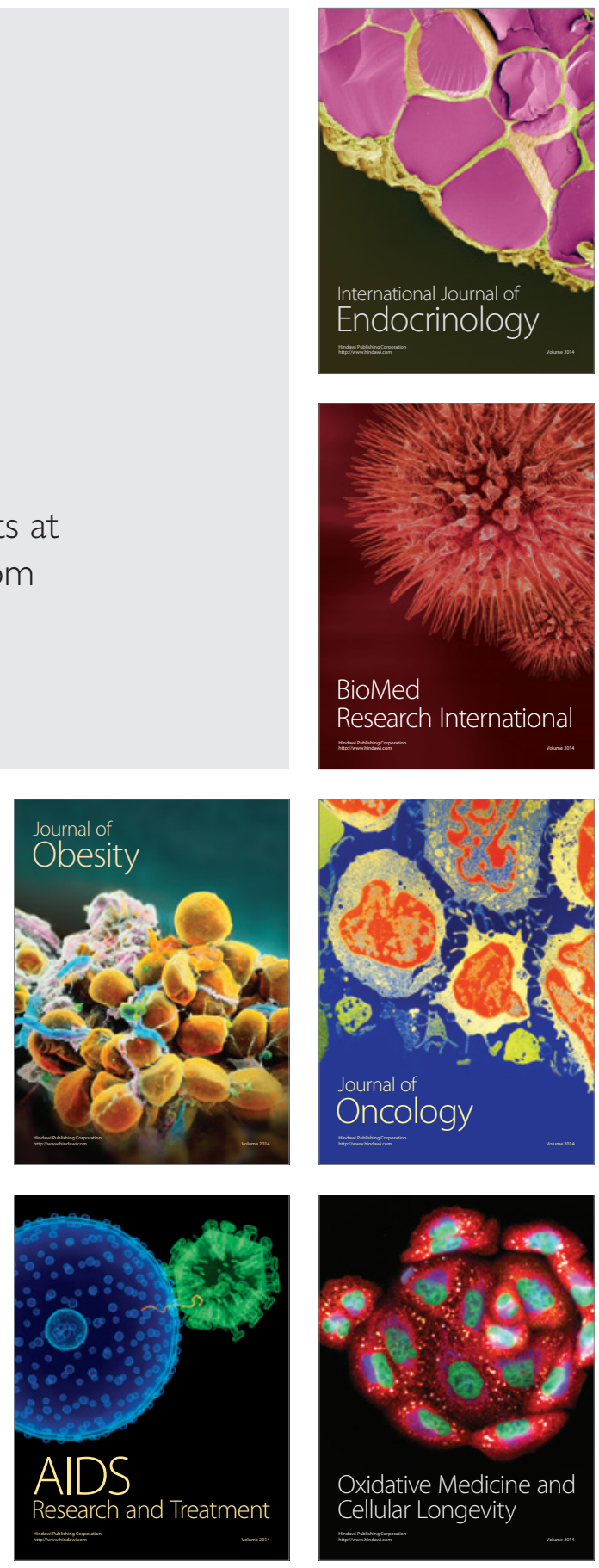